\title{
Energy Saving on Distribution of Liquid Animal Feeds at Pigsty Farms
}

Ivan Ivanovich Girutski, Andrei Grigorievich Senkov and Natalja Mikhailovna Matsveichuk

Department of Automated Production Management Systems, Belarusian State Agrarian Technical University, Minsk 220023, Belarus

\begin{abstract}
This paper focused on the task of reducing power consumption and improving energy efficiency in the technological process of distribution liquid feeds in a pigsty. The liquid feeds in a pigsty are distributed to the stables via pipelines. A centrifugal pump driven by an electric motor is used to supply the liquid feed and to move it through the pipeline. The electric motor is the major electric energy consumer in the process. As shown by experimental data and as following the theory, there is a non-linear relation between the electric motor power, the pump throughput and feed humidity. The purpose of the paper is to calculate such an optimal value of the electric motor power that provides the minimal energy consumption on the process of feed distribution. The problem is solved by the Lagrange multiplier method of nonlinear mathematical programming, taking into account geometrical parameters of the pipeline, humidity and portions volumes of distributed feed. The energy saving effect is estimated and reducing power consumption is shown.
\end{abstract}

Key words: Automation, energy saving, pigs feeding, liquid feed distribution.

\section{Introduction}

Improvement, development and implementation of efficient energy technologies and equipments in agriculture are a way to reduce the cost of agricultural products $[1,2]$. Reducing the consumption of heat and electricity can be achieved by automatic control of technological processes [1-3]. That will allow quick adjusting and optimizing the structure and parameters of the control algorithm according to real time information data about the state of the controlled biotechnical object.

Today, in the livestock industry, it is widely used technology of liquid feed distribution through pipelines [4-6]. An example of the functional scheme of such a process line is shown in Fig. 1 [7]. Liquid feed is prepared by mixing the required mass portions of dry feed and water. Then, a centrifugal pump supplies the liquid feed to the stables through the pipeline. The selector carriage successively opens and closes the faucets of the corresponding stables. The

Corresponding author: Andrei Grigorievich Senkov, Ph.D., research field: automation of technological processes. main consumer of electricity in such a technological line for distribution of liquid feed is a centrifugal pump driven by an electric motor used to supply the liquid feed and to move it through the pipeline. The hydrostatic head $H$ provided by the centrifugal pump is spent on overcoming the friction at moving the wet feed through the pipeline. The value of $H$ depends on the power of the pump motor $P(\mathrm{~kW})$ and feed humidity $W(\%)$, and determines the throughput of the pump $Q(\mathrm{~L} / \mathrm{s})$, i.e., the speed of feed distribution, as Eq. (1):

$$
P=P(Q, W)
$$

So, the reduction in energy consumption is proposed to be achieved by optimal regulation of the electric motor power and the throughput of the pump. The purpose of the paper is to calculate such an optimal value of the electric motor power that provides the minimal energy consumption on the process of feed distribution.

\section{Description}

To theoretically determine the exact mathematical 


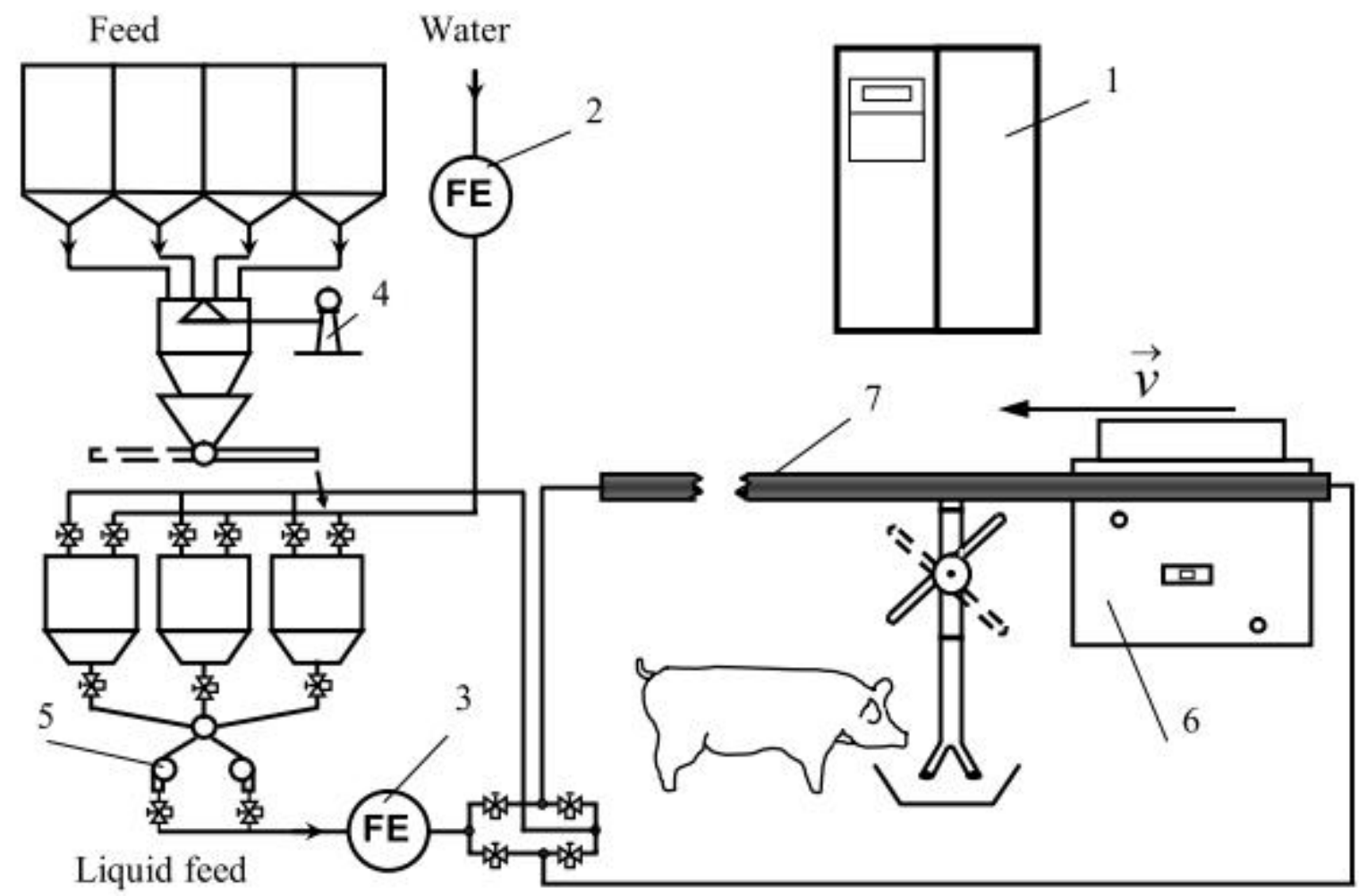

Fig. 1 Functional scheme of the process line for feeding pigs.

1-a control block with a microprocessor controller; 2-flowmeter for water; 3-flowmeter for liquid feed; 4-feed weigher; 5-a centryfugal pump supplying the liquid feed into the pipeline; 6-a selector carriage; 7 - a pipeline.

form of the relation in Eq. (1), which is a very complicated task, there should be taken into account many factors, such as geometrical parameters of the pipeline, the speed of the wet feed flow through it and the feed humidity. Therefore, in this study, the mathematical relation between power $P$ and practically important parameters of $Q$ and $W$ had been found empirically using the experimental data $[7,8]$ received by the authors and shown in Table 1 .

Since the humidity value $W$ of the feed distributed is determined by zootechnical requirements, so the real practical importance has the approximation of the relation in Eq. (1) between the pump motor power $P$ and the throughput $Q$ at any constant value of $W$. Based on Refs. [7, 8], there has been chosen the following approximating Eq. (2) for the data in Table 1:

$$
P_{W=\text { const }}=l \cdot K_{0}(W) \cdot Q^{\gamma}
$$

where, $l$ is the distance from the pump to the stable, $K_{0}$ and $\gamma$ are approximating coefficients.

Therefore, to reduce the electric energy consumption in the technological process of distribution the wet feed through the pipeline, it is necessary to control automatically the power $P$ of the electric motor used to operate the centrifugal pump. Take into account that the whole time duration of the process must be less than $t_{\max }=30 \mathrm{~min}$, which is determined by technological requirements $[7,8]$.

Assume there are $N$ stables and $i=1 \ldots N$. The authors introduce the following notation: $V_{i}$ is the amount of feed that must be supplied to stable $i(\mathrm{~L}) ; Q_{i}$ is a value of the pump's throughput at the moment of supplying the feed to stable $i(\mathrm{~L} / \mathrm{s}) ; P_{i}$ is electrical motor power of the pump at the moment of supplying the feed to stable $i(\mathrm{~kW}) ; l_{i}$ is the distance from the pump to the stable $i(\mathrm{~m})$.

According to Eq. (2), the relation between the power $P_{i}$ and throughput $Q_{i}$ is as Eq. (3): 
Table 1 Experimental data of electrical motor power $P$ depending on the pump throughput $Q$ and feed humidity $W$.

\begin{tabular}{llllllll}
\hline$W(\%)$ & & \multicolumn{7}{c}{ Corresponding $Q$ and $P$} \\
\hline \multirow{2}{*}{85.9} & $Q(\mathrm{~L} / \mathrm{s})$ & 1.28 & 1.52 & 2.25 & 2.98 & 3.63 & 3.79 \\
& $P(\mathrm{~kW})$ & 0.80 & 1.20 & 1.60 & 2.30 & 3.40 & 4.20 \\
\hline \multirow{2}{*}{84.0} & $Q(\mathrm{~L} / \mathrm{s})$ & 1.32 & 1.69 & 2.33 & 2.96 & 3.41 & 3.82 \\
& $P(\mathrm{~kW})$ & 0.60 & 1.00 & 1.60 & 2.30 & 3.30 & 4.30 \\
\hline \multirow{2}{*}{82.5} & $Q(\mathrm{~L} / \mathrm{s})$ & 1.11 & 1.74 & 2.28 & 2.63 & 3.28 & 3.56 \\
& $P(\mathrm{~kW})$ & 0.60 & 1.10 & 1.60 & 2.40 & 3.40 & 4.30 \\
\hline \multirow{2}{*}{80.0} & $Q(\mathrm{~L} / \mathrm{s})$ & 0.93 & 1.32 & 1.51 & 1.94 & 2.82 & 3.37 \\
& $P(\mathrm{~kW})$ & 0.68 & 1.10 & 1.60 & 2.40 & 3.30 & 4.30 \\
\hline \multirow{2}{*}{78.6} & $Q(\mathrm{~L} / \mathrm{s})$ & 0.44 & 0.71 & 0.92 & 1.11 & 1.47 & 2.03 \\
& $P(\mathrm{~kW})$ & 0.70 & 1.10 & 1.70 & 2.40 & 3.30 & 4.30 \\
\hline \multirow{2}{*}{77.0} & $Q(\mathrm{~L} / \mathrm{s})$ & 0.28 & 0.53 & 0.85 & 1.10 & 1.20 & 1.33 \\
& $P(\mathrm{~kW})$ & 0.70 & 1.20 & 1.80 & 2.60 & 3.50 & 4.40 \\
\hline
\end{tabular}

Table 2 The results of fitting the experimental data according to Eq. (2).

\begin{tabular}{lllllll}
\hline$W(\%)$ & 77.0 & 78.6 & 80.0 & 82.5 & 84.0 & 85.9 \\
\hline$\gamma$ & 1.12 & 1.26 & 1.42 & 1.68 & 1.78 & 1.36 \\
$K_{0}$ & 0.0210 & 0.0152 & 0.0064 & 0.0037 & 0.0030 & 0.0047 \\
\hline
\end{tabular}

$$
P_{i}=K_{0} \cdot l_{i} \cdot Q_{i}^{\gamma}
$$

where, $K_{0}$ and $\gamma$ are constant coefficients, and the numeric values of $K_{0}$ and $\gamma$ corresponding to the value of feed humidity $W$ should be taken from Table 2 .

The expression of Eq. (3) can be transformed to the following Eq. (4):

$$
Q_{i}=\left(K_{0} \cdot l_{i}\right)^{-\frac{1}{\gamma}} \cdot P_{i}^{\frac{1}{\gamma}}
$$

In this way, the time spent on supplying the required amount of feed to the stable $i$ is determined as Eq. (5):

$$
t_{i}=V_{i} / Q_{i}
$$

The energy consumption for supplying the stable $i$ with feed is determined as Eq. (6):

$$
E_{i}=P_{i} \cdot t_{i}
$$

If transform Eqs. (5) and (6) referring to Eqs. (3) and (4), then we will have the next Eq. (7):

$$
\begin{aligned}
& t_{i}=V_{i} \cdot\left(K_{0} \cdot l_{i}\right)^{\frac{1}{\gamma}} \cdot P_{i}^{-\frac{1}{\gamma}} \\
& E_{i}=P_{i} \cdot t_{i}=V_{i} \cdot\left(K_{0} \cdot l_{i}\right)^{\frac{1}{\gamma}} \cdot P_{i}^{1-\frac{1}{\gamma}}
\end{aligned}
$$

The value of the product $V_{i} \cdot\left(K_{0} \cdot l_{i}\right)^{\frac{1}{\gamma}}$ from Eq. (7) is determined by the feed humidity $W$ and the distance from the pump to the stable $i$. So, we can designate it as Eq. (8):

$$
c_{i}=V_{i} \cdot\left(K_{0} \cdot l_{i}\right)^{\frac{1}{\gamma}}
$$

Therefore, the objective function (minimization of energy consumption) takes the form of Eq. (9):

$$
E_{\Sigma}=\sum_{i=1}^{N} c_{i} \cdot P_{i}^{1-\frac{1}{\gamma}} \rightarrow \min
$$

The constraint on the maximum allowed duration time of the process is as Eq. (10):

$$
t_{\Sigma}=\sum_{i=1}^{N} c_{i} \cdot P_{i}^{-\frac{1}{\gamma}} \leq t_{\max }
$$

The constraint on the maximum allowed electric motor power is as Eq. (11):

$$
P_{i} \leq P_{\max }
$$

Let introduce a new variable expressed in Eq. (12):

$$
x_{i}=P_{i}^{-\frac{1}{\gamma}}
$$

Then, an optimization problem can be formulated as Eq. (13):

$$
\begin{aligned}
& E_{\Sigma}=\sum_{i=1}^{N} c_{i} \cdot x_{i}^{1-\gamma} \rightarrow \min , \\
& \sum_{i=1}^{N} c_{i} \cdot x_{i}-t_{\max } \leq 0 \\
& x_{\min }<x_{i}<x_{\max }
\end{aligned}
$$


So, it has a non-linear objective function and linear inequality constraints.

To solve the optimization problem in Eq. (13), the method of Lagrange multipliers [9] was used. As a result, it was found that to minimize electric energy consumption, the pump motor should run at a constant value of the power $P$ determined by Eq. (14):

$$
P^{*}=t_{\max }^{-\gamma} \cdot K_{0} \cdot\left(\sum_{i=1}^{N} V_{i} \cdot l_{i}^{\frac{1}{\gamma}}\right)^{\gamma}
$$

In that case, the electric energy consumption for distribution the whole required volume of the wet feed can be estimated by Eq. (15):

$$
E_{\Sigma}^{*}=P^{*} \cdot t_{\max }
$$

\section{Results and Discussion}

The practical importance has an estimation of the reduction of energy consumption, depending on the humidity $W$ and total volume $V_{\Sigma}=\sum_{i=1}^{N} V_{i}$ of the feed distributed at once, as these parameters are defined by zootechnical requirements and their values may vary depending on the age, weight, daily weight gain and other features of farmed animals. For example, the total volume $V_{\Sigma}$ would obviously increase with the increase of the age and live mass of the livestock. Consequently, it would increase the time duration $t_{\Sigma}$ of the feed distribution process. Similarly, the feed humidity $W$ may vary within some limits for various feeds. And the less the humidity $W$ value is, the more would be the value of $t_{\Sigma}$. So, if we assume the electric motor power $P$ as a constant value at any values of $V_{\Sigma}$ and $W$ (i.e., the reference case without the proposed calculation of optimal value $P^{*}$ by Eq. (14)), then the time duration $t_{\Sigma}$ would be the maximal at the maximum value of $V_{\Sigma}$ and the minimum one of $W$.

The initial data for the estimation are taken as the following:

$N=24$ is the total number of the stables supplied by wet feed;

$l_{0}=30$ is the distance from the pump to the 1 st stable (m);

$l=4$ is the distance between the two adjacent stables (m);

$V_{\min }=500$ is the minimum total volume of the feed distributed (L);

$V_{\max }=5,000$ is the maximum total volume of the feed distributed (L);

$t_{\max }=30$ is the maximum allowed duration time of the feed distribution process ( $\mathrm{min})$;

$W_{\min }=77 \%$ is a minimum value of the humidity $W$ of the feed;

$W_{\max }=85.9 \%$ is a maximum value of $W$.

As follows from Eq. (2) to decrease the energy consumption of the technological process, one should decrease the pump throughput $Q$. This in turn will lead to increasing the time duration $t_{\Sigma}$ of the process. But according to the technological requirements, the time $t_{\Sigma}$ in any case should be no more than $t_{\max }=30$ min. So, let consider as a reference point, such a constant value of the electric motor power $P$ of the centrifugal pump, at which a maximum total volume $V_{\max }=5,000 \mathrm{~L}$ of the feed and $W_{\min }=77 \%$ humidity, equal portions distribution into $N=24$ stables for the maximum allowed duration time of $t_{\max }=30 \mathrm{~min}$. Substituting the values of $V_{\max }, t_{\max }, l_{0}, l$ and $N$ to Eq. (14) and determining the approximating coefficients $K_{0}$ and $\gamma$ from Table 2, one can calculate $P_{\text {ref }}$ by Eq. (16):

$$
\begin{aligned}
P_{\mathrm{ref}} & =t_{\max }^{-\gamma_{(W=77 \%)}} \times K_{0(W=77 \%)} \times \\
& \left(\sum_{i=1}^{N} \frac{V_{\max }}{N} \cdot\left(l_{0}+i \cdot l\right)^{\frac{1}{\gamma_{(W=77 \%)}}}\right)^{\gamma_{(W=77 \%)}}
\end{aligned}
$$

So, we obtain a reference value: $P_{\text {ref }} \approx 5 \mathrm{~kW}$.

Considering a reference case, it is supposed that the value $P_{\text {ref }}$ is constant regardless of the values of feed total volume $V_{\Sigma}$ and humidity $W$. The choice of the reference point means that even at the worst conditions (the maximum total volume $V_{\Sigma}$ and minimum humidity $W$ ), the time duration of the process would satisfy the above mentioned constraint. Substituting the numerical value $P_{\text {ref }}$ into Eq. (7) and taking into account the data in Table 2 , the estimation 


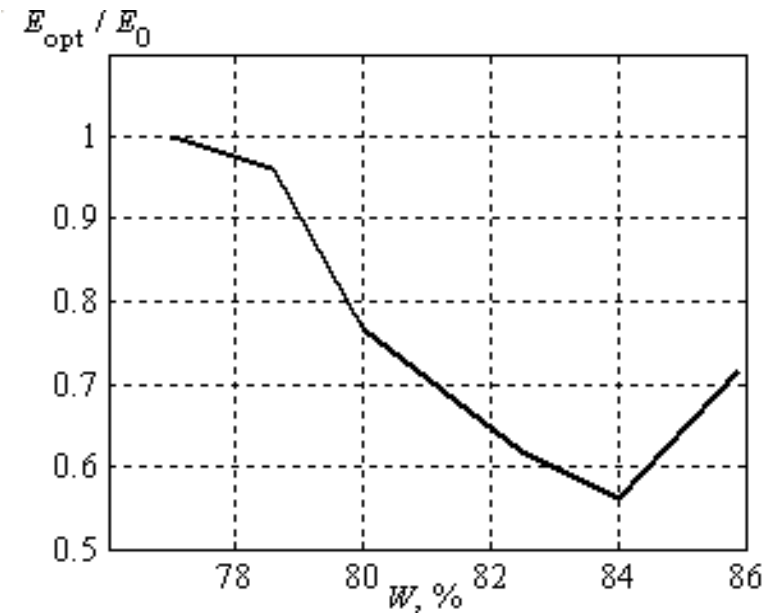

Fig. 2 Reducing the energy consumption at various values of the feed humidity $W$.

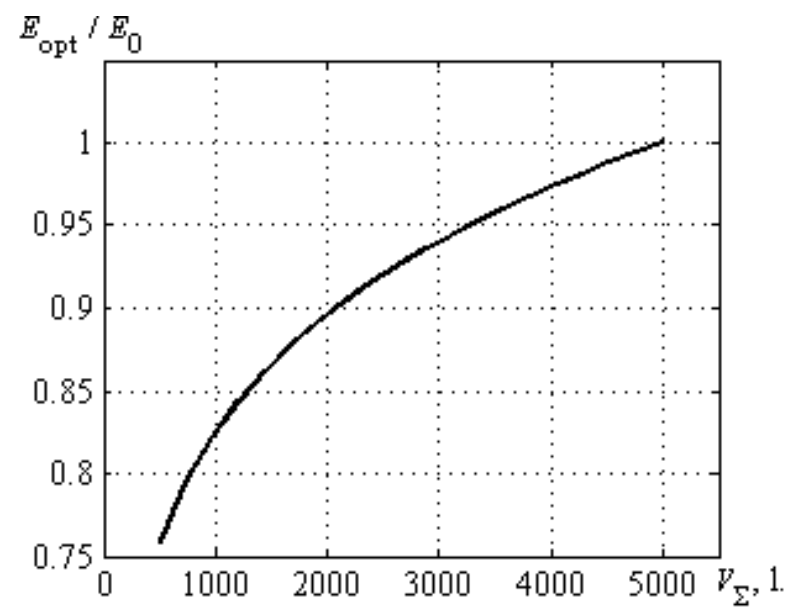

Fig. 3 Saving the energy at varoius values of feed total volume $V_{\Sigma}$.

values of the energy consumption were obtain at any values of $V_{\Sigma}$ and $W$.

Reducing the energy consumption at various values of the feed humidity $W$ is shown in Fig. 2. And saving the energy at varoius values of feed total volume $V_{\Sigma}$ is shown in Fig. 3. On both these figures, $E_{0}$ means the energy consumption of the process at the reference point and $E_{\text {opt }}$ means the energy consumption of the process optimized by the proposed method.

Thus, as one can see from the Figs. 2 and 3, the proposed method of optimal power control of the pump motor takes into account geometric parameters of the pipeline, the spatial arrangement of the stables, the feed humidity and total volume, and provides reduction in energy consumption for the technological process of distribution of wet feed. Depending on the conditions of the liquid feed, the energy saving effect may vary from $0 \%$ up to $40 \%$ for a single act of feed distribution.

\section{Conclusions}

This paper solved the problem of reducing the energy consumption for the process of liquid feed distribution through pipelines at pigsty farms. The proposed optimization formula calculates an optimal power value of the electric motor of centrifugal pump taking into account geometrical parameters of the pipeline, humidity and portions volumes of distributed feed, and provides the minimum total energy consumption on the process. The energy saving effect is estimated and reducing power consumption up to $40 \%$ is shown.

\section{References}

[1] EUR-Lex. 2006. "Directive 2006/32/EC of the European Parliament and of the Council of 5 April 2006 on Energy End-Use Efficiency and Energy Services and Repealing Council Directive 93/76/EEC." OJ L 114. Accessed April 27, 2006. http://eur-lex.europa.eu/legal-content/EN/TXT /?uri=uriserv:OJ.L_.2006.114.01.0064.01.ENG\&toc $=$ OJ: L:2006:114:TOC.

[2] Teagasc-The Agriculture and Food Development Authority. 2011. Energy Use in Agriculture. Oak Park, Carlow: Teagasc Head Office, 88.

[3] Gellings, C. W., and Parmenter, K. E. 2004. "Energy Efficiency in Fertilizer Production and Use." In Efficient Use and Conservation of Energy, edited by Gellings, C. W., and Blok, K. Oxford, UK: Encyclopedia of Life Support Systems (EOLSS) Publishers.

[4] Beaudoin, G. 1998. Stall Manager System, Module and Feeding Device for Management of Meal Times and Food Distribution in Animal Stalls. US Patent 5,816,191, Filed May 15, 1996, and Issued October 6, 1998.

[5] Forster, M. 2001. Method and Apparatus for Providing Domestic Animals with Liquid Food. US Patent 6,205,952, Filed October 6, 1999, and Issued March 27, 2001.

[6] Voogd, L. E. N., and Van Den Berg, K. 2003. Device for Automatically Supplying a Predetermined Amount of Least One Sort of Feed to an Animal in a Predetermined Period. US Patent 6,619,228, Filed October 2, 2002, and Issued September 16, 2003. 
[7] Girutski, I. I. 2008. Threading Mechanized Technological Lines for Distribution the Pig Feed with Microprocessor Control. Moscow, Russia: Russian State Agrarian University, Moscow Timiryazev Agricultural Academy, 36.

[8] Girutski, I. I., Matsveichuk, N. M., and Senkov, A. G.
2015. "Power Saving Opportunities of Intellectual Control of Distributing of Liquid Animal Feeds at Pigsty Growing Farms." Mechanization in Agriculture 10: 12-5.

[9] Bertsekas, D. P. 1999. Nonlinear Programming, 2nd ed.. Cambridge, MA: Athena Scientific. 